\title{
Sitzung vom 13. December 1880.
}

Vorsitzender: Hr. A. W. Hofmann, Vice-Präsident.

Der Vorsitzende beklagt, dass er die traurige Pflicht zu erfüllen habe, der Gesellschaft den Tod zweier ausgezcicbneter Fachgenossen anzuzeigen.

Am 24. November habe die Gesellschaft ihr Ehrenmitglied Sir Benjamin Collins Brodie verloren, dessen Name an eine Reihe der interessantesten chemischen Forscbungen erinnere. Den älteren Fachgenossen brauche er kaum in's Gedächtniss zurückzurufen, dass es Brodie gewesen sei, welcher durch eine meisterhafte Jugendarbeit zuerst Licht über die Gruppe der Wachskörper verbreitet habe. Die Ansichten über diese Verbindungen, welche aus Brodie's Arbeiten hervorgegangen, seien die noch heute geltenden. Spätere Mittheilungen desselben bezögen sich auf die verschiedenen Modificationen des Schwefels, auf das Verbalten des Jods zum Phosphor, auf die Zersetzungen des Bariumsuperoxyds, welche letztere Untersuchung ibn bekanntlich zur Entdeckung der Superoxyde der organischen Radicale führte. Es sei Brodie's Scharfblick gewesen, welcher zuerst den Unterschied des gebundenen Aethyls und des freien Aethyls klar gelegt, und auf diese Weise die lange Discussion über Alkobol und Aether, welche durch Williamson's Forschungen nahezu bis zur Spruchreife gediehen war, zum Abschluss gebracbt habe. Noch wolle er an die grosse Untersuchung Brodie's über den Graphit erinnern, an die Bestimmung des Atomgewichts des Graphits, an die Entdeckung der Graphitsäure. Die Auffindung einer Methode der chemischen Disintegration des Graphits habe ihm den lebhaften Uank der Industrie eingetragen in einer Zeit, in welcher die Erschöpfung der Graphitlager von Borrowdale die Fabrikation in neve Bahnen gedrängt habe. Allerdings habe die beinahe unmittelbar darauf folgende Entdeckung der grossen Graphitlager in Sibirien, sowie in Nordamerika, die Ver- 
werthung der Brodie'schen Disintegrationsmethode unnöthig gemacht, allein die Erscheinung bleibe immer eine der bemerkenswerthesten, and es gebe wobl keinen Cursus der Mineralchemie, in welchem der Brodie'sche Versuch nicht angestellt werde. Endlich gedenke auch Jeder noch der schönen Untersuchungen über das Ozon; sei doch Odling's Hypothese, dass das Moleculargewicht des Ozons andertbalb Mal so gross sei, als das des Sauerstoffs, welche allerdings auch schon durch Soret wabrscheinlich gemacht worden war, gleichwohl erst durch Brodie's Versuche endgültig festgestellt worden. Bemerkenswerth sei die Originalität einer jeden der Brodie'schen Untersuchungen; jede derselben schlage ganz eigentlich einen neuen Ton an. Es sei hier richt der Ort in die Einzelheiten dieser Forschungen einzugehen, noch weniger aber der zahlreichen und umfassenden theoretischen Speculationen zu gederken, in denen sich Brodie mit Vorliebe bewegt habe. Zweifle er doch nicht daran, dass die Gesellschaft bald in der Lage sein werde, ihrem dahingeschiedenen Ehrenmitgliede ein Denkmal der Erinnerung zu setzen. Unter den zahlreichen englischen Freunden Brodie's seien Viele, die ein anziehendes Lebensbild des Mannes zu geben rermöchten, und er denke zumal an Einen, der mit dem Heimgegangenen die Pfade der Jugend gewandelt, der mit ihm zu Füssen Liebig's gesessen, der auch später in stetem, freundschaftlichem Verkebr mit ihm gestanden habe, mithin über Brodie's ganzen Lebenslauf zu berichten vermöge. Er werde uns die Zeit schildern können, in welcher Brodie als Faraday's College in der Royal Institution wirkte, seine Lehrthätigkeit an der Universität Oxford, endlich seinen beschaulichen Lebensabend unter den schattigen Laubgängen seines Landsitzes in Surrey, wo Brodie von der Welt zurückgezogen, nur noch seiner Familie, seinen Freunden und der Wissenschaft lebte. Und Niemand werde diese Lebensskizze aus der Haud legen, der nicht neben der Bewunderung für den ausgezeicbneten Gelebrten auch die wohlthuende Frinnerung an den liebenswärdigen Charakter des edien Mannes in sich aufgenommen hätte.

Noch, fährt der Vorsitzende fort, müsse er leider der Versammlung einen zweiten schweren Verlust anzeigen, welchen die Wissenschaft erlitten habe. Am 1. December sei Professor Wilbelm Heintz in Halle von einer kurzen scbweren Krankheit dahin gerafft worden. Heintz babe der Gesellschaft nicht näher angebört, allein das sei kein Grund, weshalb er nicht an dieser Stelle aussprechen solle, wie hoch die wissenschaftlichen Verdienste des Verstorbenen ron den Fachgenossen angeschlagen würden, wie sehr ihm seine Freunde zugethan gewesen seien, wie aufrichtige Verehrung auch die ihm weniger Nabestehenden seinem edlen Cbaracter gezollt hätten. Die Begabung des Mannes sei eine sehr vielseitige gewesen, seine Interessen hätten 
sich nach den verschiedensten Richtungen bin verzweigt. Obwobl sich seine Thätigkeit in späterer Zeit mit Vorliebe auf dem Gebiete der organischen Chemie concentrirt habe, so läge doch auch eine ganze Reibe von Arbeiten über Gegenstände der anorganischen, der analytischen und der physiologischen Chemie oder mineralogischen Inhalts von ihm vor. Auch für die physikalischen Disciplinen habe Heintz ein lebhaftes Interesse gehegt und ein dankbares Andenken werde ihm zumal auch in einer hiesigen Schwestergesellschaft, in der physikalischen Gesellschaft bewahrt, welche er vor mebr als dreissig Jahren in Gemeinschaft mit Beetz, Brücke, du Bois-Reymond, Karsten und $\mathrm{Knobla}$ uch gegründet habe. Sollten aus der grossen Anzahl von Arbeiten, welche wir Heintz verdanken, einige wenige besonders nambaft gemacht werden, so würde wohl ein Jeder zunächst an seine langjährige Untersuchungen über die Fettkörper denken, deren umfassende Mannichfaltigkeit am anschaulichsten aus der in der Mitte der fünfziger Jahre von ihm veröffentlichten Znsammenstellung dieser Arbeiten bervortrete. Die zwischen der Palmitinsäure und Stearinsäure liegende Säure, welche man lange Zeit Margarinsäure genannt habe, sei zuerst von Heintz synthetisch dargestellt worden. Das Studium der Einwirkung der Monochloressigsäure auf Alkohole und Phenole have zur Erkenntniss grosser Klassen never und höchst interessanter Körper geführt; ebenso fruchtbar seien seine umfassenden Untersuchungen über das Verbalten dieser Säure zom Ammoniak gewesen, aus denen der organischen Chemie die Kenntniss der Diamido- und Triamidosäuren zugewachsen sei. Die schwierige und noch nicht vollständig zum Abschluss gekommene Arbeit über die Acetonamine sei noch frisch in der Erinnerung der Anwesenden. Noch wolle er bier eine frühere Beobachtung besonders berrorbeben. Heintz sei es gewesen, der zuerst den Uebergang des Chloroforms, unter dem Einflusse des Ammoniaks bei hoher Temperatur, in Blausäure beobachtet babe; er fühle sich um so mehr gedrungen, diese wichtige Entdeckung Heintz zu vindiciren, da er selber (Redner) sie irrthümlich ror einiger Zeit einem anderen Forscher zugeschrieben habe ${ }^{1}$ ). Diese flüchtigen Bemerkungen hätten keinen anderen $Z$ weck als den, an einige der hervorragendsten Forschungen zu erinnern, welche die Wissenschaft $\mathrm{Heintz}$

1) In meiner Abhandlung: Ueber eine neue Reihe von Isomeren der Nitrile (Monatsber, d. Berl. Akad. 1867, 651) heisst es: "In einer Porlesung wollte ich meinen Zuhörern die merkwürdige und im Sinne moderner Anschauungsweisen so lehrreichen Bildung der Blauslure aus Ammoniak und Cbloroform zeigen, welche $\mathrm{Hr}$. Cloëz kennen gelehrt hat." Bei dieser Citation hatte jch die von Hra. Cloëz im Jahre 1858 (Compt. Rend. XLVI, 785) veröffentlichte Abbandlung im Sinne. Erst später bin ich darauf aufmerksam gemacht worden, dass die Beobachtung des Uebergangs des Chloroforms in Blausäure von Heintz schon viel fruber, ntimlich im Jabre 1856 (Ann. Chem. Pharm. C, 369) gensacht worden ist.

A. W. H. 
verdanke; er dürfe sich aber in Kürze fassen, da er bereits im Stande sei, der Gesellschaft eine eingehende Schilderung des Lebens und der wissenschaftlichen Thätigkeit des Dahingeschiedenen aus berufenster Feder in Aussicht zu stellen. Fin ausgezeichneter Schüler des Verstorbenen, Hr. Wislicenus, habe sich bereits in pietätvoller Freundschaft dieser Aufgabe gewidmet und dem von ihm zu gebenden Lebensbilde werde Hr. E. du Bois Reymond ein Blatt der Erinnerung an seinen Jugendfreund beifügen.

Die Versammlung erhebt sich ron ihren Sitzen, um das Andenken der Geschiedenen zu ehren.

Nach Genehmigung des Protocolls der letzten Sitzung werden zu ausserordentlichen Mitgliedern proclamirt die Herren:

Ludolf Lichtenstein, Leipzig;

A If red Sarauw, Zürich;

Dr. Paul Schoop, Zürich;

Dr. H. B. Dettmer, Bonn;

A. Fr. Palfy, Erkner;

V. J. Stephan, Libochowitz;

Ide Lint, Leiden;

Paul Friedrich Frische,

Alexander Neubaus,

Ferdinand Regelsberger,

Ferdinand Bergmann,

Würzburg;

Richard Möckel,

Leon Schröter,

Carl Schwedes,

Hans Kobek,

$\left.\begin{array}{l}\text { Robert Ludwig, } \\ \text { Benno Sandmann, }\end{array}\right\}$ Berlin;

Dr. ron Reiss, Aachen.

Zu ausserordentlichen Mitgliedern werden vorgeschlagen die Herren:

Alexander Claparède, Univers.-Laborat., Bonn (durch O. Wallach und L. Claisen);

Vit torio Serravallo, Magister der Pharmacie, Rom (durch G. Ciamician und M. Dennstädt);

D. Orme Masson, University College, Bristol (durch Sydney Marsden und S. Gabriel); 
Ph. Ott, Berlin, Seydelstrasse 21 (durch Ferd. Tiemann und W. Will);

Dr. Adolf Döring, Professor an der Universität Cordoba (durch C. Rammelsberg und C. Liebermann);

H. N. S tokes, John Hopkins University, Baltimore (durch Ira Remsen und N. Morse);

Carl Piest, Auguststr. 31, Berlin, / (durch F. Tiemann Heinrich Rehberg, Grossbeeren- ( und E. Baumann); strasse 5, Berlin,

G. Viereck, Göttingen (durch B. Tollens und H. Wichelhaus);

W. W. Nicol M. A., F. R. S. E., Edinburgh, 15. Blacket Place (durch Sydney Marsden und S. Gabriel);

Jobnson, Lieutenant D. D., Berlin W., Behrenstr. 39 (durch A. W. Hofmann and S. Gabriel);

Boleslaw Ebert, Berlin, Hegelplatz 2 (durch Julius Philipp und J. Natanson).

Für die Bibliothek sind als Geschenke eingegangen:

90. Wiadomości farmaceutyczne, Tom VII, 10 a. 11 .

26. v. Fehling, H. Neues Handwörterbuch der Chemie. Band III. Liefrg. 10. (Vom Herausgeber.)

869. Muck, F. Ueber ein Mineralvorkommen auf der Zeche Courl in Westfalen. (Verf.)

1092. Benjamin, M. Sugar analysis. New York 1880. (Verf.)

1093. Bötsch, K. Ueber das Verhalten einiger Harze bei der Destillation uber Zinkstaub. - Zur Kenntniss der Saligeninderivate. Sep.-Abdr. (Verf.)

1094. Cech, C. O. Zur Kenntniss der Zersetzungsproducte in faulenden Hübnerei. I. Mitth. - Zur Kenntniss des Kaffeeöls. - Ueber den Farbstoff des Rubus Chamaemorus. - Ueber desinficirende Wirkungen der Chlorpbenole.

1095. - - Oenochemische Untersuchungen russischer Reben und Fruchtweine. (In russischer Sprache.) St. Petersburg 1880, Sep.-Abdr. (Verf.)

1096. Hof, Adolf. Beitrigge zur Kenntniss des Phenanthrenchinons. Inang-Diss. Marburg 1880. (Verf.)

1097. Kinch, Edward. Contributions of the agricultural chemistry of Japan. Sep. Abdr. (Verf.)

870. Hofmann, A. W. Die Frage der Theilung der philosophischen Facultat. (Rectoratarede.) Berlin 1880.

Der Schriftführer
A. Pinner.
A. W. $\mathrm{H}$ of $\mathrm{m}$ a $\mathrm{n} \mathrm{n}$.

Der Vorsitzende 